\title{
Usability and the multimedia industry: the Australian Multimedia Testing Centre
}

\author{
Clark N. Quinn \& Malcolm Raymond \\ Access Australia Cooperative Multimedia Centre \\ Australian Technology Park \\ Cornwallis Street \\ Eveleigh NSW 1430 \\ Australia \\ clarkq@cmcaccess.com.au
}

\begin{abstract}
The Australian Multimedia Testing Centre performs usability evaluation to support the multimedia industry. The Centre is equipped with an industry-standard usability laboratory including a testing room, video recording and mixing, and an observation room. The Centre takes a client-centred approach, providing usability evaluation consulting services and a variety of options for testing. A free initial consultation is offered to establish an evaluation plan which meets the client's time frame, scope of project, and budget. In addition, the Centre is pro-actively sponsoring research to increase the understanding of the specific issues of usability for multimedia, including cognitive and affective issues.
\end{abstract}

KEY WORDS usability, evaluation, multimedia

\section{INTRODUCTION}

The Australian Multimedia Testing Centre (AMTC) was established in September 1996 by Access Australia Cooperative Multimedia Centre. Access Australia is one of six such centres established under an initiative of the Commonwealth government with a mission to support the multimedia industry through collaboration between industry partners and cultural and educational institutions. The AMTC is one of several avenues through which Access Australia is supporting multimedia development.

The AMTC offers a range of test services appropriate to the Australian multimedia industry. Initial services are centred in the areas of performance and usability testing, implemented through laboratory facilities dedicated to each. Performance testing of multimedia products includes evaluating whether specified functionality is met, how the product handles under stress conditions including low memory and storage, compatibility testing across vendor platforms, and testing for performance with different configurations.

In order to ensure provision of the complete suite of services required by the industry, the Centre is assessing the need for and viability of other testing services, including educational evaluation of learning products and marketability analysis. This report specifically addresses the usability activities of the AMTC.

\section{THE LAB}

The usability lab consists of one fixed and one portable facility. The fixed installation was designed to be practical yet at a very affordable price and was based on the recommendations made in a study commissioned by Access Australia.

The fixed lab uses a typical usability set-up with one room to contain the evaluator (typical end user) engaged in the task, and another room for observers who are making an assessment of usability issues occuring in the cvaluation scssion. In addition, an operator controls the video mixing equipment to combine images of the screen and the user. The two rooms are separated by a one way mirror so that the evaluators are not immediately aware of the operator.

The practice of usability testing arises from the AMTC policies.

\section{POLICY}

The AMTC necessarily takes a broad view of what constitutes multimedia, including CDROM product as well as World Wide Web-based (WWW) projects. The AMTC is equipped through the performance laboratory with a vast array of different hardware and software on which to conduct testing. The AMTC is also provided with internet server capability for WWW tests.

The AMTC is committed to the utmost quality in practice, and is in the process of ISO 9000 certification 
to that end. Quality procedures ensure regular review of the methodology. The AMTC's partners play a major role in the quality of the usability evaluation method.

\section{PARTNERS}

In order to ensure practical services and to ensure that the results of research are applied in an appropriate manner, AMTC has established a relationship with Performance Technologies, a usability consultation firm. Performance Technologies provides the usability evaluation methodology used at the Centre.

The AMTC has also partnered with the Human Computer Interaction (HCI) Group of the Commonwealth Science and Industry Research Organisation's (CSIRO) Division of Information Technology. The $\mathrm{HCl}$ Group has a quality audit role ensuring that the services of the Centre are of the highest standard and that techniques and methodologies used are appropriate and reflect the latest understanding of usability testing for multimedia products. The $\mathrm{HCI}$ Group of CSIRO also plays a role with the AMTC to define new usability requirements.

Access Australia, in support of the multimedia industry also provides education and training. Access Australia and the AMTC are working with the Hiser Group, and the other partners, to develop usability training.

\section{RESEARCH}

Usability issues with multimedia products can the very different to those encountered in more traditional task oriented computer systems. In particular, there are a whole series of new usability issues to consider in Web-based environments. In order to provide a better understanding of usability issues of multimedia projects the AMTC has initiated an ongoing research program with the $\mathrm{HCl}$ Group of CSIRO.

A steering committee with representatives from Access Australia, the AMTC, CSIRO's HCI Group, and Performance Technologies, consults on the research directions. In collaborative research, the AMTC and the $\mathrm{HCl}$ Group seck to identify the ways in which usability is affected by multiple media, as wetl as the changes that occur when the goal of the product is education, entertainment, or both.

The results of multimedia usability research will also be disseminated through Access Australia education and training.

\section{OUTCOMES}

The consequences of this commitment to quality are already being scen. In its first few months the Centre has already successfully tested both CDROM and WWW products. The results of evaluation are not just provided to the client, but a subset of the projects are sclected to be case studies to evaluate the process Client satisfaction has been evidenced in both word of mouth recommendations and repeat business. 